\title{
Vibratory Somatosensory Stimulus Improves Neuromechanical Task of Stair Climbing by the Elderly
}

\author{
Seunghun Ko, ${ }^{1 \dagger}$ Kiyoung Kwak, ${ }^{2 \dagger}$ Mattatika Pomrop, ${ }^{1}$ and Dongwook Kim ${ }^{2,3 *}$ \\ ${ }^{1}$ Department of Healthcare Engineering, Graduate School, Jeonbuk National University, \\ Jeonju 54896, Republic of Korea \\ ${ }^{2}$ Division of Biomedical Engineering, College of Engineering, Jeonbuk National University, \\ Jeonju 54896, Republic of Korea \\ ${ }^{3}$ Research Center for Healthcare \& Welfare Instrument for the Elderly, Jeonbuk National University, \\ Jeonju 54986, Republic of Korea
}

(Received August 27, 2020; accepted April 22, 2021)

Keywords: local vibration, mu rhythm, stair climbing, biomechanics, elderly

The purpose of this study was to investigate the influence of sensorimotor-cortex-responsebased vibratory stimulation on stair climbing by the elderly and to reveal the functional assistance effect of vibratory stimulation. To do this, vibratory stimulation conditions (VSCs) were set through the reduction of the mu rhythm, an index of sensorimotor cortex excitability, and applied to elderly people during stair climbing. Joint angle, moment, power, and muscle coactivation (CA) of lower limbs were analyzed to investigate the influence of VSCs on stair climbing. As a result of vibratory stimulation, the biomechanical characteristics of the ankle and hip joints were further developed according to the VSC, and a tendency to compensate the knee joint work appeared. Also, functional improvement of the ankle joint was shown through greater energy transfer from the knee joint to the ankle joint. These results suggested that sensorimotorcortex-response-based vibratory stimulation has the effect of functional assistance of the movement of elderly people and may be useful in research on movement assistance or rehabilitation for people with reduced body functions.

\section{Introduction}

Stair climbing is an important motion for mobility and is a functional task that is repeatedly required in everyday life. It is a complex and difficult movement in which the flexion and extension of the lower limb segments are repeated periodically. ${ }^{(1-3)}$ Stair climbing involves different muscles, forces, and body movements from level walking, ${ }^{(3,4)}$ and the functional demands on the musculoskeletal system are high due to the need for a larger range of motion, high muscle moment, and weight loading at the three joints of the lower limbs. ${ }^{(5-8)}$ In particular, the contact force in the knee joint is on average three times the body weight and can even reach six times the body weight, ${ }^{(2)}$ and stair climbing requires a higher flexion moment than level walking and has been reported to be a difficult task in terms of biomechanics. ${ }^{(3)}$

\footnotetext{
*Corresponding author: e-mail: biomed@jbnu.ac.kr

†Seunghun Ko and Kiyoung Kwak contributed equally to this work as co-first author. https://doi.org/10.18494/SAM.2021.3354
} 
Stair climbing is a more difficult task for elderly people, whose musculoskeletal system is weakened by aging. Previous studies ${ }^{(5,9)}$ showed that when climbing stairs, young adults have high flexion and moments in the knee and ankle joints, whereas elderly people have less flexion in the knee and ankle joints and more in the hip joint. Samuel et al. ${ }^{(10)}$ confirmed that higher hip joint flexion and knee joint extensor moments appear in elderly people during stair climbing. In particular, it has been reported that the knee joint flexor moment during stair climbing is about three times greater than that of level walking. ${ }^{(4)}$ It has been reported that elderly people have high functional demands of the hip and knee joints during stair climbing, and the levels of demand leave little reserve capacity for them to draw on in unexpected situations or circumstances. $^{(10,11)}$ That is, elderly people perform motion with limited physical ability, and as a result, they cannot cope with unexpected situations or circumstances, and have difficulty with limb control, body balance, and stability control during motion. Therefore, it is necessary to reduce the biomechanical demands required for motion execution by assisting the motion of elderly people.

Local vibratory stimulation can be used to assist motion. Previous studies reported on the positive effects of local vibratory stimulation on the musculoskeletal system, such as increased joint flexibility, ${ }^{(12-14)}$ improved postural control, ${ }^{(15)}$ improved muscle performance, ${ }^{(16)}$ and increased muscle activity. ${ }^{(17,18)}$ Specifically, Lapole and Pérot ${ }^{(12)}$ confirmed that it is possible to reduce muscle-tendon stiffness and increase joint flexibility through vibrations repeatedly applied to the Achilles tendon. Rothmuller and Cafarelli ${ }^{(19)}$ reported that vibratory stimulation applied to the patella tendon during voluntary knee extension can improve the co-contraction of the hamstring.

In addition, vibratory stimulation applied to the limbs affects the sensorimotor cortex through afferent fibers, which appears as a decrease in mu rhythm. ${ }^{(20)} \mathrm{Mu}$ rhythm is a kind of electroencephalogram (EEG) that consists of 8-13 Hz activities that appear maximally over the sensorimotor cortex and is used as an index of excitability in the sensorimotor cortex due to the attenuation of its characteristics by tactile stimulation and vibration. ${ }^{(12,20-24)}$ Previous studies have reported that local vibratory stimulation affects the sensorimotor cortex ${ }^{(12,21,22)}$ and the excitability of the corticospinal tract. ${ }^{(23,24)}$ Furthermore, it was found that there is an inversely linear correlation between the mu rhythm and force in the sensorimotor cortex during isometric contraction, ${ }^{(24)}$ and it was reported that a change in motor-evoked potential (MEP) appeared due to local vibration. ${ }^{(25)}$ In particular, changes in afferent fibers and MEPs that are activated according to vibration characteristics have been reported. ${ }^{(16)}$

Therefore, in this study, we set up vibratory stimulation conditions (VSCs) based on the sensorimotor cortex response to investigate the neuromechanical effects of VSCs on the stair climbing task of elderly people. For this, the VSCs were set by investigating the mu rhythm excitability for each stimulated site and vibration frequency of each subject. Then, the neuromechanical changes in elderly people based on the applied VSC during the stair climbing were investigated. Finally, the main effects of vibratory stimulation and the mechanism that induces the neuromechanical changes are discussed. 


\section{Materials and Methods}

\subsection{Subjects}

In this study, 10 elderly males (age: $76.0 \pm 1.7$ years, height: $166.7 \pm 3.7 \mathrm{~cm}$, weight: $68.0 \pm$ $5.5 \mathrm{~kg}$ ) and 15 young males (age: $25.5 \pm 1.5$ years, height: $173.2 \pm 2.6 \mathrm{~cm}$, weight: $72.4 \pm 4.4 \mathrm{~kg}$ ) participated as test and control groups, respectively. It was confirmed that no subjects had experienced diseases of the musculoskeletal and nervous systems of the lower limbs or surgery. All subjects gave their written informed consent before participation in the study after receiving a sufficient explanation of the experiment, such as the experimental environment and procedure. One day before the experiment, to exclude factors that may affect the experiment, the subjects were asked to adequately rest and to refrain from drinking alcohol and excessive exercise. At least one hour before the EEG measurement experiment, all subjects were asked to refrain from caffeine intake and smoking, which may have otherwise affected the EEG signal. This research was approved by the Institutional Review Board (IRB) of Jeonbuk National University (IRB File No. JBNU 2017-03-011-001).

\subsection{Setting of VSCs}

\subsubsection{Vibration perception threshold measurement}

The vibration perception threshold for each tendon of the subjects was investigated to set the vibration intensity. A small linear actuator (DMJBRN0934AA; Samsung Electro-Mechanics Co., Ltd., Korea) and a function generator (AFG-21 25; Good Will Instrument Co., Ltd., Taiwan) were used to apply local vibratory stimulation to the tibialis anterior tendon (TAT), Achilles tendon (ACT), quadriceps tendon (QT), and biceps femoris tendon (BT), which can activate the tibial anterior muscle (TA), triceps surae muscle, quadriceps femoris muscle, and biceps femoris muscle (BF), respectively. The target vibration frequency range was the $100-300 \mathrm{~Hz}$ band, in which the peripheral nerve system reacts sensitively. In the vibration threshold measurement, the amplitude was gradually increased, and the moment when the subject first recognized the sense of vibration was recorded. Three measurements were made for each frequency, and the average value was set as the vibration perception threshold.

\subsubsection{EEG recording according to vibration characteristics}

EEG signals were collected to analyze the central nervous system response according to the vibratory perception threshold and vibration frequency, and the mu rhythm in the sensorimotor cortex was analyzed. EEG disk electrodes were attached to positions $\mathrm{C} 3, \mathrm{Cz}, \mathrm{C} 4, \mathrm{FC} 3, \mathrm{FCz}, \mathrm{FC} 4$, $\mathrm{CP} 3, \mathrm{CPz}$, and $\mathrm{CP} 4$ corresponding to the sensorimotor areas according to the international 10-10 system, and the reference electrodes were placed on both earlobes. A vision recorder (Brain Products GmbH, German) was used for EEG measurement, and measurements were performed at nine frequencies and with no stimulus (None) excluding frequencies $(100,120,260,280$, $300 \mathrm{~Hz}$ ) at which some subjects did not feel any vibration in a previously performed vibration 
perception threshold measurement. The experiment was conducted in a soundproof dark room, and the subjects maintained a comfortable state with their eyes closed. EEGs were measured for 1 min per frequency, and a 5 min break was provided between the vibration stimuli to prevent adaptation to the vibration sensation.

\subsubsection{Analysis of mu rhythm change for vibration characteristics}

BESA software (BESA GmbH, Germany) was used to analyze the mu rhythm. The measured EEG signal was filtered at $0.1-60 \mathrm{~Hz}$ using a bandpass filter. Each EEG recorded for 1 min was divided into 30 epochs by defining $2 \mathrm{~s}$ as one epoch, and the mu rhythm ratio in the total EEG spectrum for each epoch was calculated using power spectrum density analysis. The amount of mu rhythm reduction was calculated by comparing None and the mu rhythm during vibration stimulation. The VSCs were set based on the vibration perception threshold and mu rhythm reduction.

\subsection{Stair climbing experiment}

\subsubsection{Stair climbing protocol}

Three-dimensional kinematic and kinetic data were collected using the Optotrak Certus system (NDI Inc., Canada), which is a three-dimensional marker-based optical motion capture system considered as a standard of motion analysis. ${ }^{(26)}$ Three Optotrak cameras were placed around the stairs, and four force plates (Bertec Inc., USA) were embedded in the footpath and the third step of the stair. Infrared-emitting diode markers were attached to the 11 major areas of the subject's lower limbs. First Principles software (Northern Digital Inc., Canada) was used to record the markers and the ground reaction force data.

Stair climbing was performed using a five-step wooden staircase with each step having a raise of $16 \mathrm{~cm}$, a tread of $30 \mathrm{~cm}$, and a width of $180 \mathrm{~cm}$ (Fig. 1). Subjects were asked to climb the stairs at their self-selected velocity in a step-over-step manner, and before the experiment, subjects performed several test trials to familiarize themselves with the stairs.

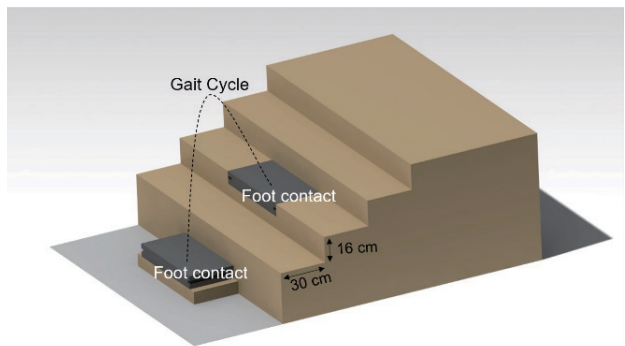

(a)

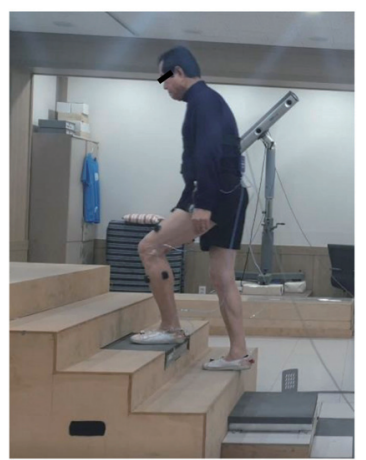

(b)

Fig. 1. (Color online) Stair climbing experiment: (a) model of staircase and (b) example of stair climbing. 
Stair climbing was performed three times for each VSC including None. The electromyogram (EMG), motion capture, and ground reaction force were measured at the start of stair climbing, and vibratory stimulation was applied at the same time. A 3 min break was provided between each VSC to prevent subjects from adapting to the vibration sensation.

\subsubsection{Motion analysis of stair climbing}

SIMM (MusculoGrapics Inc., USA) was used to analyze the biomechanical characteristics of stair climbing in elderly people and the changes according to the VSC. The gait cycle in stair climbing was defined as the motion from the foot contact of the third step to the foot contact of the same leg on the fifth step. To reduce the variability between subjects, the angle, moment, and power of the three joints were normalized according to the defined gait cycle, and the moment and power were also normalized by the weight of each subject. The stair climbing motion was analyzed by dividing it into weight acceptance (WA, 0-14\%), pull-up (PU, 14-32\%), forward continuance (FCN, 32-64\%), foot clearance (FC, 64-82\%), and foot placement (FP, 82-100\%) in reference to the study of McFadyen and Winter. ${ }^{(7)}$ For the angle, moment, and power, the average profile of three trials was obtained for the three joints, and the ensemble average profile of each VSC was obtained to compare the difference between VSCs.

\subsubsection{EMG recording and signal processing for $\mathrm{CA}$}

The EMG signal was measured to calculate muscle CA during stair climbing. A Trigno ${ }^{\mathrm{TM}}$ Wireless EMG system (Delsys Inc., USA) was used, and surface EMG sensors were placed over TA, gastrocnemius lateralis muscle (GL), gastrocnemius medialis muscle (GM), soleus muscle (Sol), vastus lateralis muscle (VL), vastus medialis muscle (VM), RF, and BF, and attached to the muscles, with the attachment locations based on the SENIAM proposal (Fig. 2). ${ }^{(27)}$

EMG signal processing was performed using EMGworks analysis (Delsys Inc., USA) and MATLAB R2018b (Mathworks Inc., USA). The raw EMG signal was resampled to $1000 \mathrm{~Hz}$ and

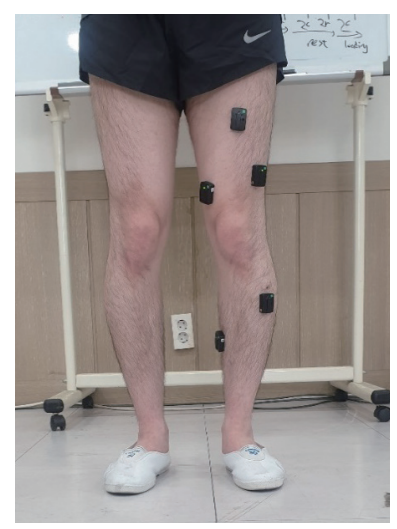

(a)

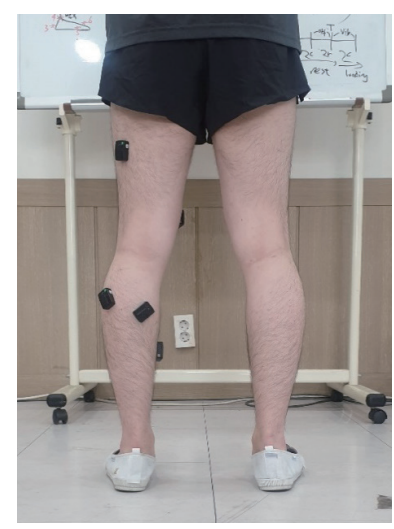

(b)

Fig. 2. (Color online) EMG sensor locations: (a) front of leg and (b) back of leg. 
rectified and filtered with a cut-off frequency of $6 \mathrm{~Hz}$ by a fourth-order Butterworth low-pass filter. EMG amplitude was normalized to the maximum peak of each trial and time-normalized according to the gait cycle of stair climbing. The average EMG profile of the standardized EMG signal measured three times for each VSC was obtained, and the ensemble average of each VSC was obtained using the average EMG of all subjects.

\subsubsection{Muscle co-activation (CA) analysis}

CA was calculated as follows using the normalized EMG signal of each trial and the method proposed by Falconer and Winter:(28)

$$
\text { Co-activation }=2 \times\left\{\text { Common Area }\left(M_{(a)} \& M_{(b)}\right) /\left(M_{(a)} \text { Area }+M_{(b)} \text { Area }\right)\right\} \times 100 .
$$

$\boldsymbol{M}_{(\boldsymbol{a})}$ and $\boldsymbol{M}_{(\boldsymbol{b})}$ represent the two muscles in the agonist-antagonist muscle relationship. $\boldsymbol{M}_{(\boldsymbol{a})}$ Area represents the area under the EMG graph of $\boldsymbol{M}_{(\boldsymbol{a})}$, and $\boldsymbol{M}_{(b)}$ Area represents the area under the EMG graph of $\boldsymbol{M}_{(b)}$. Common Area represents the area where the graphs of $\boldsymbol{M}_{(\boldsymbol{a})}$ and $\boldsymbol{M}_{(\boldsymbol{b})}$ overlap. ${ }^{(27)}$

CA was calculated for TA-GL, TA-GM, TA-Sol, VL-BF, VM-BF, and RF-BF of the agonistantagonist muscle relationship. The average CA of the VSC in each muscle group was calculated, and the ensemble average of each muscle group was calculated using the average CA of all subjects.

\subsection{Statistical analysis}

SPSS 20 (IBM SPSS Statistics, USA) was used for statistical analysis. A paired t-test was performed to determine the statistical significance of the difference between the mu rhythm of None and that of the vibration stimuli. An independent t-test was performed to analyze the statistical significance of the difference between the two groups during stair climbing, and the statistical significance was defined as $p<0.05$. The Friedman test was used to confirm the statistical significance of the angle, moment, power, and CA according to the VSC. Post hoc analyses were performed using the Wilcoxon signed-rank test and statistical significance was defined as $p<0.05$.

\section{Results}

\subsection{Mu rhythm change according to vibration frequency}

Figure 3 shows the change in the mu rhythm of each tendon with the vibration frequency. The frequencies at which the greatest mu rhythm reduction in each tendon were shown were $140 \mathrm{~Hz}$ for ACT, $240 \mathrm{~Hz}$ for TAT, $240 \mathrm{~Hz}$ for QT, and $160 \mathrm{~Hz}$ for BT.

We set stimulation frequencies of 180 and $190 \mathrm{~Hz}$, values at which the participants reacted sensitively in previous studies as found using sensory receptors, ${ }^{(29,30)}$ and $250 \mathrm{~Hz}$, at which a 


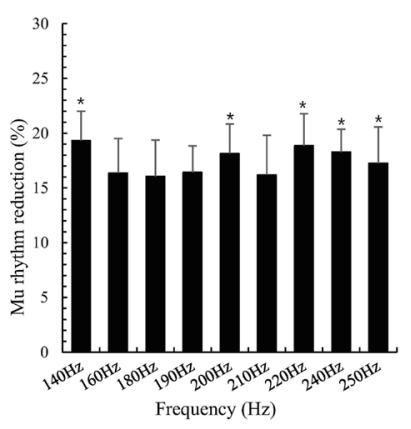

(A) ACT

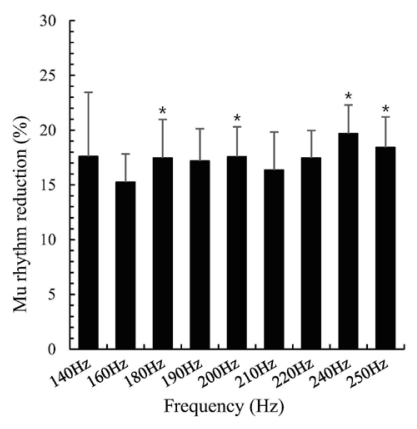

(B) TAT

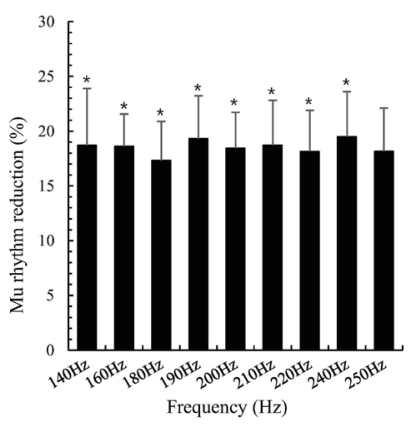

(C) QT

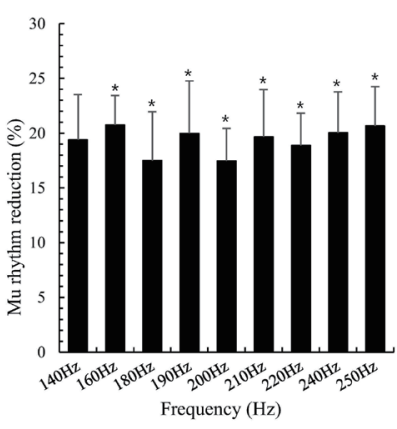

(D) BT

Fig. 3. Mu rhythm reduction according to local vibratory stimulation frequency. ${ }^{*} p<0.05$, i.e., a statistical difference between None and the vibratory stimulation frequency: (A) ACT, (B) TAT, (C) QT, and (D) BT.

rapid increase in the threshold value was observed in this study, as single-frequency VSCs (applied equally to all tendons). In addition, the following frequencies producing the maximum excitability of the sensorimotor cortex in this study were combined as a multifrequency stimulation (MF) condition: ACT: $140 \mathrm{~Hz}$, TAT: $240 \mathrm{~Hz}$, QT: $240 \mathrm{~Hz}, \mathrm{BT}$ : $160 \mathrm{~Hz}$. In this way, a total of four VSCs were set and applied during stair climbing.

\subsection{Kinetic and kinematic profiles in stair climbing}

\subsubsection{Angle, moment, and power of elderly according to VSC}

The angle, moment, and power of None and each VSC for the young and elderly people during stair climbing are shown in Fig. 4, and Table 1 shows the values and statistical significance of the peaks for each VSC. Elderly people showed a decrease in the knee extensor moment peak (Knee EMp1) and $(+)$ power peak (Knee Pp) of WA-PU and an increase in the knee flexion peak (Knee Ap) and knee extensor moment peak (Knee EMp2) during FCN. Among the VSCs, the most effective frequency was $180 \mathrm{~Hz}(p<0.05)$. The hip joint showed an increase in the flexion peak (Hip Ap), extensor moment peak (Hip EMp), and (+) power peak (Hip Pp) in WA-PU, and a decrease in the hip flexion peak (Hip Ap2) in FC-FP $(p<0.05)$. Among the VSCs, MF for WA-PU and $250 \mathrm{~Hz}$ for FC-FP showed the greatest effect $(p<0.05)$. The ankle joint showed an increased plantar-flexor moment peak (Ankle PMp1) in WA-PU, and an increased dorsiflexion peak (Ankle Ap1, Ap2), plantar-flexor moment peak (Ankle PMp2), and $(+)$ power (Ankle Pp) under all VSCs during FCN. Among the VSCs, $250 \mathrm{~Hz}$ and MF of WA-PU and $180 \mathrm{~Hz}$ of FCN showed significant effects $(p<0.05)$.

\subsubsection{Muscle CA according to VSC}

Table 2 shows the changes in CA for each muscle group in elderly people according to the VSC during stair climbing. CA decreased in all groups of the lower leg (TA-GL, -GM, -Sol) 


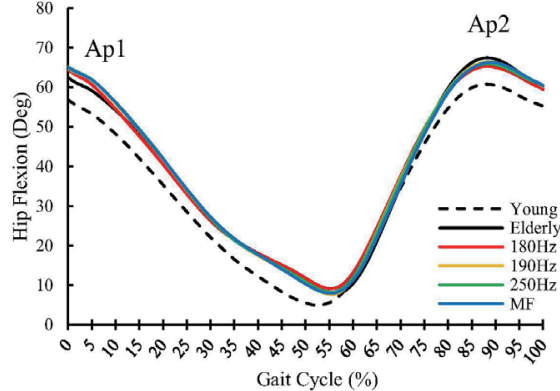

(a)

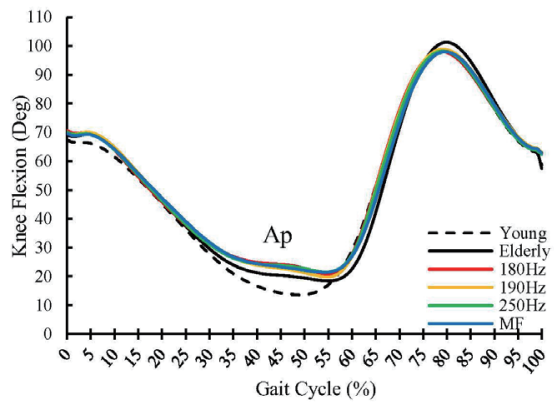

(d)

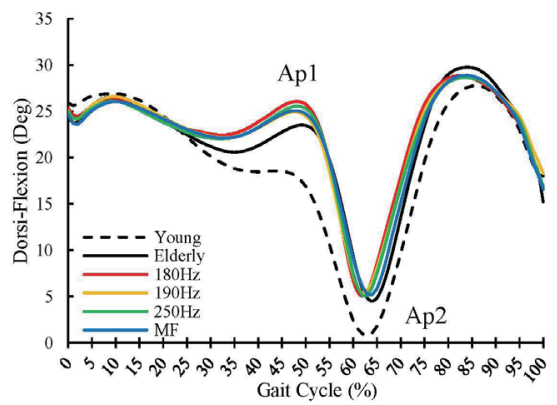

(g)

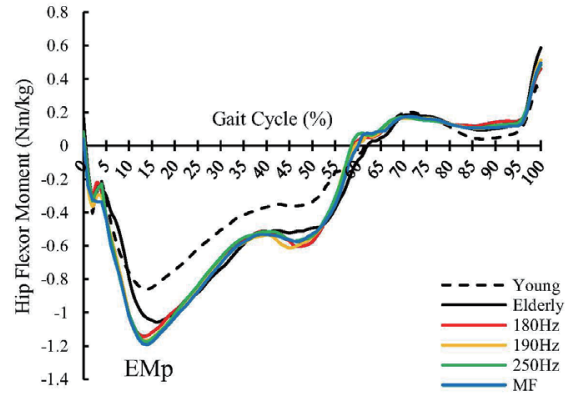

(b)

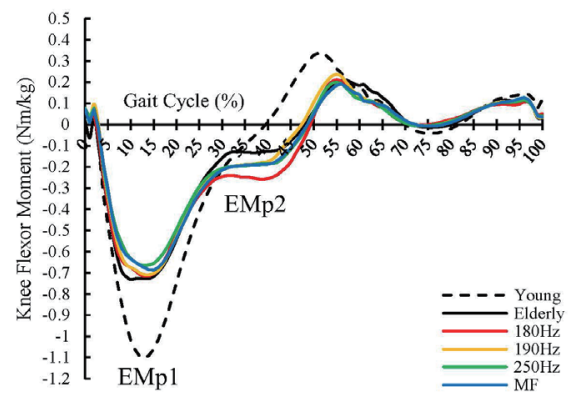

(e)

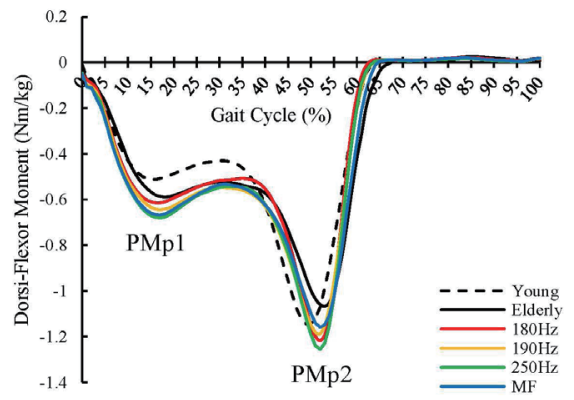

(h)

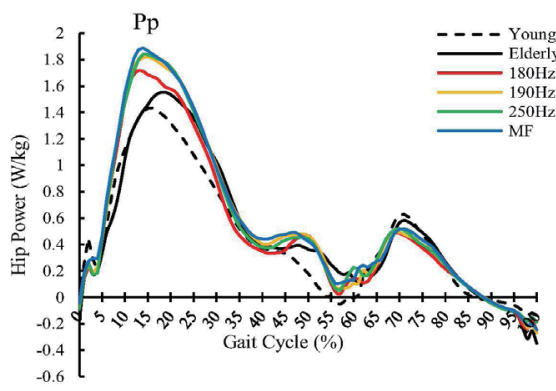

(c)

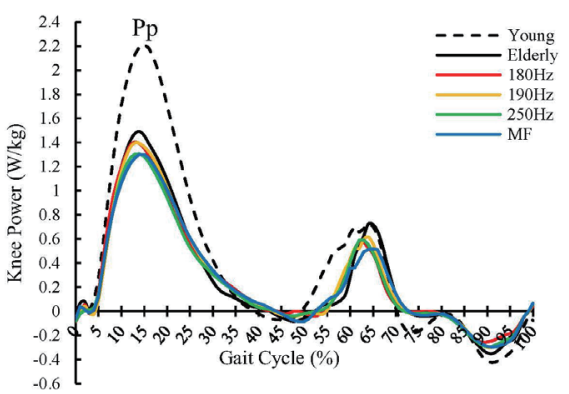

(f)

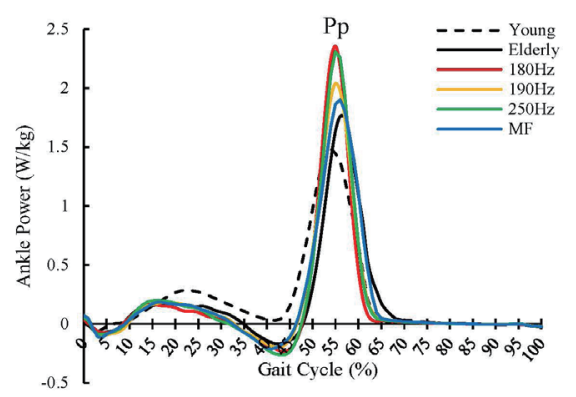

(i)

Fig. 4. (Color online) Kinematic and kinetic profiles of stair climbing in young (control) and elderly: angle peak (Ap), extensor moment peak (EMp), plantar-flexor moment peak (PMp), and power peak (Pp): (a) Hip flexion, (b) Hip flexor moment, (c) Hip joint power, (d) Knee flexion, (e) Knee flexor moment, (f) Knee joint power, (g) Dorsiflexion, (h) Dorsiflexor moment, and (i) Ankle joint power.

under all conditions, and statistical significance was confirmed under all conditions except for $250 \mathrm{~Hz}$ for TA-GL and $190 \mathrm{~Hz}$ and MF for TA-Sol $(p<0.05)$. All groups had an overall tendency of decreased CA in the thigh. For HAM-VL and HAM-VM, CA decreased under all conditions except $180 \mathrm{~Hz}$, and CA was lowest for $190 \mathrm{~Hz}(p<0.05)$. CA for the HAM-RF group decreased at 180 and $190 \mathrm{~Hz}$ and the decrease was significant at $190 \mathrm{~Hz}(p<0.05)$. 
Table 1

Peak value ( \pm standard deviation) of angle, moment, and power of young and elderly during stair climbing.

\begin{tabular}{lcccccc}
\hline \multirow{2}{*}{ Parameter } & \multicolumn{5}{c}{ None } & \multicolumn{5}{c}{ VSCs of elderly } \\
\cline { 2 - 6 } Hip & Young & Elderly & $180 \mathrm{~Hz}$ & $190 \mathrm{~Hz}$ & $250 \mathrm{~Hz}$ & MF \\
\hline Ap1 & $56.86 \pm 3.53^{\dagger}$ & $62.55 \pm 2.61$ & $64.52 \pm 2.68$ & $64.77 \pm 1.86$ & $65.17 \pm 1.74^{*}$ & $64.99 \pm 1.54^{*}$ \\
Ap2 & $60.77 \pm 2.93^{\dagger}$ & $67.49 \pm 2.44$ & $65.31 \pm 2.37^{*}$ & $66.37 \pm 1.80$ & $65.96 \pm 1.93^{*}$ & $66.31 \pm 1.90$ \\
EMp & $0.849 \pm 0.07^{\dagger}$ & $-1.033 \pm 0.085$ & $-1.143 \pm 0.093$ & $-1.165 \pm 0.076$ & $-1.176 \pm 0.068^{*}$ & $-1.193 \pm 0.078^{*}$ \\
Pp & $1.435 \pm 0.21$ & $1.414 \pm 0.228$ & $1.718 \pm 0.234^{*}$ & $1.814 \pm 0.166^{*}$ & $1.842 \pm 0.191$ & $1.887 \pm 0.180^{*}$ \\
\hline Knee & \multicolumn{7}{c}{} & & \\
Ap & $13.60 \pm 1.73^{\dagger}$ & $36.82 \pm 6.42$ & $45.40 \pm 7.42^{*}$ & $44.68 \pm 6.37^{*}$ & $44.15 \pm 8.23^{*}$ & $41.56 \pm 7.36$ \\
EMp1 & $1.103 \pm 0.10^{\dagger}$ & $-0.732 \pm 0.121$ & $-0.715 \pm 0.145$ & $-0.710 \pm 0.125$ & $-0.666 \pm 0.124$ & $-0.686 \pm 0.131$ \\
EMp2 & $0.137 \pm 0.08^{\dagger}$ & $-0.134 \pm 0.109$ & $-0.258 \pm 0.089^{*}$ & $-0.195 \pm 0.081$ & $-0.197 \pm 0.080$ & $-0.196 \pm 0.070$ \\
Pp & $2.196 \pm 0.23^{\dagger}$ & $1.491 \pm 0.316$ & $1.403 \pm 0.269$ & $1.397 \pm 0.223$ & $1.306 \pm 0.247$ & $1.301 \pm 0.244$ \\
\hline Ankle & \multicolumn{7}{c}{} & \\
\hline Ap1 & $19.23 \pm 1.70^{\dagger}$ & $23.52 \pm 2.53$ & $26.06 \pm 1.13^{*}$ & $24.94 \pm 1.74$ & $25.56 \pm 1.83^{*}$ & $25.08 \pm 1.51$ \\
Ap2 & $0.80 \pm 2.89^{\dagger}$ & $4.87 \pm 1.69$ & $8.52 \pm 1.92^{*}$ & $8.12 \pm 2.24$ & $7.49 \pm 2.75^{*}$ & $5.85 \pm 2.32$ \\
PMp1 & $0.512 \pm 0.10$ & $-0.589 \pm 0.078$ & $-0.613 \pm 0.088$ & $-0.643 \pm 0.050$ & $-0.680 \pm 0.081^{*}$ & $-0.668 \pm 0.066^{*}$ \\
PMp2 & $1.146 \pm 0.11$ & $-1.066 \pm 0.086$ & $-1.216 \pm 0.032^{*}$ & $-1.186 \pm 0.062$ & $-1.255 \pm 0.049^{*}$ & $-1.159 \pm 0.060$ \\
Pp & $1.486 \pm 0.38$ & $1.760 \pm 0.407$ & $2.357 \pm 0.210^{*}$ & $2.040 \pm 0.341$ & $2.309 \pm 0.303^{*}$ & $1.901 \pm 0.237$ \\
\hline
\end{tabular}

$\dagger p<0.05$. Young (control) vs Elderly, marked only in Young

$* p<0.05$. Statistical significance in stimulus compared to None

Ap: Angle peak, EMp: Extensor moment peak, PMp: Plantar-flexor moment peak, Pp: Power peak, MF: Multifrequency stimulation (ACT: $140 \mathrm{~Hz}$, TAT: $240 \mathrm{~Hz}$, QT: $240 \mathrm{~Hz}, \mathrm{BT}: 160 \mathrm{~Hz}$ )

Table 2

Mean value ( \pm standard deviation) of None and VSC during stair climbing.

\begin{tabular}{lccccccc}
\hline \multicolumn{7}{c}{ Agonist-antagonist muscle CA } \\
\hline \multirow{2}{*}{ None } & TA-GL & TA-GM & TA-Sol & HAM-VL & HAM-VM & HAM-RF \\
\hline \multirow{4}{*}{ VSC of elderly } & Young & $50.3 \pm 5.1$ & $44.3 \pm 4.6^{\dagger}$ & $41.8 \pm 3.7^{\dagger}$ & $67.9 \pm 1.8^{\dagger}$ & $67.6 \pm 1.8^{\dagger}$ & $68.8 \pm 1.7^{\dagger}$ \\
& Elderly & $48.2 \pm 5.6$ & $50.7 \pm 6.2$ & $49.6 \pm 6.1$ & $73.3 \pm 4.2$ & $74.3 \pm 4.3$ & $75.0 \pm 2.9$ \\
\hline & $180 \mathrm{~Hz}$ & $42.5 \pm 8.1^{*}$ & $45.1 \pm 7.3^{*}$ & $45.2 \pm 7.3^{*}$ & $74.1 \pm 1.9$ & $75.0 \pm 3.1$ & $74.1 \pm 2.7$ \\
& $190 \mathrm{~Hz}$ & $42.5 \pm 5.5^{*}$ & $46.1 \pm 5.9^{*}$ & $48.3 \pm 6.8$ & $69.5 \pm 3.3^{*}$ & $70.2 \pm 4.2^{*}$ & $72.5 \pm 3.3^{*}$ \\
& $250 \mathrm{~Hz}$ & $42.6 \pm 8.6$ & $43.2 \pm 8.3^{*}$ & $44.8 \pm 8.5^{*}$ & $72.5 \pm 3.5$ & $73.7 \pm 3.7$ & $76.3 \pm 4.1$ \\
& $\mathrm{MF}$ & $45.3 \pm 7.8$ & $45.1 \pm 7.0^{*}$ & $47.9 \pm 8.0$ & $73.0 \pm 2.6$ & $74.2 \pm 2.4$ & $75.7 \pm 2.4$ \\
\hline
\end{tabular}

${ }^{\dagger} p<0.05$. Young (control) vs Elderly, marked only in Young

${ }^{*} p<0.05$. Non-stimulus vs vibratory stimuli

MF: Multifrequency stimulation (ACT: $140 \mathrm{~Hz}, \mathrm{TAT}: 240 \mathrm{~Hz}$, QT: $240 \mathrm{~Hz}, \mathrm{BT}: 160 \mathrm{~Hz})$

\section{Discussion}

\subsection{Effects of vibratory stimulation on sensorimotor cortex}

To set the VSC based on the sensorimotor cortex response, vibratory stimulation was applied to each tendon, and the mu rhythm reduction according to the vibration frequency was examined. As a result, it was confirmed that the mu rhythm was reduced by applying vibratory stimulation. Furthermore, frequencies showing a particularly high decrease in each tendon were identified. This may be because the degree of vibration perception depends on the vibratory stimulation site. The distribution density of sensory receptors differs according to the body part, ${ }^{(31,32)}$ and 
the vibration frequency that can be detected varies, resulting in a difference in information transmitted to the sensorimotor cortex. In previous studies, it was confirmed that the excitability of the cerebral cortex ${ }^{(33)}$ and the MEP are increased by vibration stimulation. ${ }^{(34,35)}$ This means an increase in the excitability of the motoneuron pool, ${ }^{(36)}$ which represents a change in the number of motor neurons in response to vibratory stimulation. ${ }^{(37)}$ Therefore, the mu rhythm reduction in this study shows that the vibration applied to the tendon influenced the excitability of the sensorimotor cortex, which will affect the excitability of the motoneuron pool and muscle activity.

\subsection{Changes in biomechanics of joints of elderly people under vibratory stimulation}

The biomechanical characteristics of elderly people include reduced high knee joint demand and increased hip and ankle joint demand, and local vibratory stimulation further develops the characteristics of the hip and ankle joints to compensate for the work of the knee joint.

Knee EMp1 and Knee Pp in WA-PU of elderly people were lower than those of young adults, and they decreased with vibratory stimulation. This is a strategy for maintaining a reserve moment and is considered a safe strategy for elderly people with limited physical ability performing motion as it reduces the load on the knee joint. ${ }^{(11)}$ The reduced Knee EMp1 and Knee Pp due to vibratory stimulation is a result of the reduction of the knee joint extensor work, and it is beneficial in maintaining joint moment reserves. At this time, the work of the reduced knee joint is compensated by the extensor activity of the hip and ankle joints and will contribute to the increased safety of elderly people. ${ }^{(11)}$ Also, a decrease in the knee joint extensor activity is indicated by a decrease in the thigh muscle CA. The reduced CA means that joint freedom can be ensured by reducing the joint stiffness and more flexible motion can be performed. Furthermore, knee mobility for the standing alignment in the stance phase is ensured. During FCN, Knee Ap and Knee EMp2 of elderly people are higher than those of young adults, and knee EMp2 is prolonged [Fig. 4(e)]. This means a delay in the extension of the knee joint, which can be confirmed through the knee angle (knee Ap) at that time. Through this delay in the joint extension, a high extensor moment can be generated and energy can be transferred to the ankle. ${ }^{(11)}$ During FCN, the increased Knee Ap and EMp2 under vibratory stimulation means that the knee joint is in a more flexed state, creating a larger extensor moment and delivering more energy to the ankle joint. The transferred energy helps maintain dorsiflexion of the ankle joint.

The ankle joint has a higher PMp1 for elderly people than for young adults in WA-PU and is increased by vibratory stimulation. This may be because the plantar-flexor muscle of the ankle joint compensates for the knee joint moment, which is reduced by the moment reserve strategy. The ankle joint in elderly people has a higher Ap1 and Ap2, lower PMp1, and higher Pp than those in young adults during FCN. At that time, the leg prepares to toe-off to climb to the next step, and the GL and GM as the plantar-flexor muscle of the ankle joint display concentric contraction. The lower ankle joint moment in elderly people may be caused by the ankle's proximity to the center of rotation, and it will cause the joint moment to remain within the joint limits. ${ }^{(38)}$ Also, the $(+)$ power (ankle Pp) is higher in elderly people than in young adults because of the energy transmitted from the knee to the ankle through GL and GM. ${ }^{(11)}$ The transmitted 
energy helps maintain dorsiflexion and contributes to toe-off to raise the body when climbing stairs. The increased ankle Ap1, Ap2, PMp2, and Pp are due to the vibratory stimulation during FCN. This is due to the increase in the knee Ap and EMp2, which will contribute to maintaining the dorsiflexion and toe-off of the ankle joint by producing more energy and transferring more energy to the ankle joint through extension delay. Also, the muscle CA of the lower leg is decreased by local vibratory stimulation (Table 2). The reduced CA means that the flexion transition of the ankle joint becomes easier during the stance phase by enabling joint freedom.

The hip joints of the elderly people have higher Ap1, Ap2, EMp, and Pp than those of the young adults in WA-PU (Table 1). After foot landing, the leg is extended to accept the weight, and the hip joint lifts the body. The increased angle, moment, and power of the hip joint are intended to compensate for the decreased knee joint demand along with the increase in the low ankle PMp1. The Knee EMp and Pp, increased by vibratory stimulation, work together with the ankle joint extensor to compensate for the work of the knee joint extensor. The reduced thigh CA contributes to the extension of the hip and knee joints while standing by increasing the joint freedom.

In other words, the vibratory stimulation effect on stair climbing in elderly people is a mechanism that compensates the work of the knee joint by further developing the biomechanical characteristics of the hip and ankle joints. This reduces the high load and demand of the knee joint, which are characteristics of stair climbing. The hip and ankle joints compensate for the work of the knee joint in the early stance phase by local vibratory stimulation, and the greater knee flexion and moment during FCN result in more energy transferred to the ankle through the extension delay, thus achieving a functional improvement of the ankle joint. Thus, the VSC that showed the greatest effect was MF of WA-PU and $180 \mathrm{~Hz}$ of FCN.

\subsection{Mechanism of functional local vibratory stimulation for motion assistance of elderly people}

When local vibratory stimulation was applied to stair climbing, a neuromechanical change appeared, and the change depended on the applied vibration characteristics. This mechanical change is due to the variation in the input of the afferent nerve fibers based on the VSC applied to the lower limbs, which may be due to the influence on the central pattern generator (CPG). The CPG is a neural network in which interconnected excitatory and inhibitory neurons generate rhythmic patterns without sensory input, and it is presumed to be located in the brain stem or the entire spinal cord and plays an important role in motion such as walking and swimming. ${ }^{(39)}$ The CPG responds flexibly to sensory input and is modified by information from peripheral receptors and signals from other areas of the central nervous system. ${ }^{(40)}$ In previous studies, changes in the CPG due to the input of Ia, Ib afferent fibers during locomotion were confirmed, ${ }^{(39)}$ and it was reported that activation of the lumbar CPG is possible through peripheral nerve stimulation or muscle vibration. ${ }^{(39)}$ Moreover, Steyvers et al. ${ }^{(24)}$ reported that MEP was altered by local vibratory stimulation. MEP changes are due to the operation of cortical circuits or cortical responses. ${ }^{(37)}$ Information on local vibratory stimulation is transmitted to the cerebrum and cerebellum through afferent nerve fibers, and the CPG is controlled through activities related to the sensorimotor cortex and cause a change in motion. 
The MF was effective at the start phase of the stair climbing, and the single-frequency stimulation showed its main effect at the subsequent phase of stair climbing. This difference may be due to the conscious intervention required to perform the start phase. To climb stairs, it is first necessary to recognize and control the position of the foot at the beginning of the motion. Hortobágyi and DeVita ${ }^{(41)}$ confirmed that when elderly people step downward, they contact the ground with limbs straightened out as opposed to young adults, indicating that a conscious effort is made to adjust the position of the limb before it touches the ground. Also, Shin et al. ${ }^{(42)}$ reported that elderly people have increased dependence on visual information for maintaining posture control when stair walking.

In summary, stair climbing requires the conscious mediation of lower limb movements to ensure positional stability and posture control at the start phase of stair climbing, resulting in more involvement of the central nervous system and more sensitive responses to MF. Since the subsequent phase of the motion is performed according to the basic rhythmic pattern of the CPG, it should respond more quickly to single-frequency stimulation.

\section{Conclusions}

We investigated the sensorimotor cortex response under local vibration stimulation and, on this basis, VSCs were set. Furthermore, we analyzed the neuromechanical changes that appeared when applying different VSCs to elderly people when stair climbing. We obtained the following conclusions.

First, it was confirmed that the mu rhythm decreased in response to vibration stimulation. The amount of reduction depended on the vibration frequency and stimulation site, and the vibration frequency showing a particularly high reduction in each tendon was confirmed. Second, the biomechanical characteristics of the hip and ankle joints were developed to compensate for the work of the knee joint, and the ankle joint was functionally improved through energy transfer from the knee joint to the ankle joint. Third, MF at the early phase of stair climbing and single-frequency stimulation at the subsequent phase showed major effects. It is probable that at the early phase of the stair climbing, the movement of the lower limbs was expressed through conscious intervention to ensure posture control and positional stability, and subsequent movement was performed according to the rhythmic pattern of the CPG programmed by the central nervous system.

These results suggest that sensorimotor-cortex-response-based local vibratory stimulation is effective in assisting the movement of elderly people, and the effect depends on the characteristics of the vibratory stimulation. This shows that the sequence of motion and biomechanical patterns should be considered. These results may be helpful in the field of research related to movement performance of elderly people or the rehabilitation for people with reduced body functions.

\section{Acknowledgments}

This work was supported by National Research Foundation of Korea (NRF) grants funded by the Korean government (MSIT) (NRF-2017R1A2B2009389 and NRF-2019R1A2C2088033), and 
by the Basic Science Research Program through the National Research Foundation of Korea (NRF) funded by the Ministry of Education (NRF-2019R1A6A3A01092848). This research was also supported by "Research Base Construction Fund Support Program" funded by Jeonbuk National University in 2020.

\section{References}

1 R. Riener, M. Rabuffett, and C. Frigo: Gait. Posture 15 (2002) 32. https://doi.org/10.1016/S09666362(01)00162-X

2 P. A. Costigan, K. J. Deluzio, and U. P. Wyss: Gait. Posture 16 (2002) 31. https://doi.org/10.1016/S09666362(01)00201-6

3 A. Protopapadaki, W. I. Drechsler, M. C. Cramp, F. J. Coutts, and O. M. Scott: Clin. Biomech. 22 (2007) 203. https://doi.org/10.1016/j.clinbiomech.2006.09.010

4 T. P. Andriacchi, G. B. Andersson, R. W. Fermie, D. Stern, and J. O. Galante: J. Bone. Joint. Surg. Am. 62 (1980) 749.

5 W. R. Taylor, M. O. Heller, G. Bergmann, and G. N. Duda: J. Orthop. Res. 22 (2004) 625. https://doi. org/10.1016/j.orthres.2003.09.003

6 C. Steele Ed.: Applications of EMG in Clinical and Sports Medicine (InTech, London, 2012) pp. 117-130.

7 B. J. McFadyen and D. A. Winter: J. Biomech. 21 (1988) 733. https://doi.org/10.1016/0021-9290(88)90282-5

8 Y. C. Lin, L. A. Fok, A. G. Schache, and M. G. Pandy: J. Biomech. 48 (2015) 340. https://doi.org/10.1016/j. jbiomech.2014.11.019

9 K. Karamanidis, A. Arampatzis: Ann. Biomed. Eng. 37 (2009) 467. https://doi.org/10.1007/s10439-008-9624-7

10 D. Samuel, P. Rowe, V. Hood, and A. Nicol: Gait. Posture 34 (2011) 239. https://doi.org/10.1016/j. gaitpost.2011.05.005Get

11 N. D. Reeves, M. Spanjaard, A. A. Mohagheghi, V. Baltzopoulos, and C. N. Maganaris: J. Electromyogr. Kinesiol. 19 (2009) 57. https://doi.org/10.1016/j.jelekin.2007.09.009

12 T. Lapole and C. Pérot: J. Electromyogr. Kinesiol. 21 (2011) 87. https://doi.org/10.1016/j.jelekin.2010.10.011

13 A. M. Kinser, M. W. Ramsey, H. S. O’Bryant, A. A. Christopher, A. S. William, and H. S. Michael: Med. Sci. Sports. Exerc. 40 (2007) 133. https://doi.org/10.1249/mss.0b013e3181586b13

14 V. B. Issurin, D. G. Liebermann, and G. Tenenbaum: J. Sports. Sci. 12 (1994) 561. https://doi. org/10.1080/02640419408732206

15 A. Karimi-AhmadAbadi, S. Naghdi, N. N. Ansari, Z. Fakhari, and M. Khalifeloo: J. Bodyw. Mov. Ther. 22 (2018) 42. https://doi.org/10.1016/j.jbmt.2017.04.013

16 B. P. Couto, H. R. Silva, M. P. Barbosa, and L. A. Szmuchrowski: Int. J. Sports. Med. 33 (2012) 123. https://doi. org $/ 10.1055 / \mathrm{s}-0031-1286294$

17 J. Luo, B. P. McNamara, and K. Moran: Med. Eng. Phys. 27 (2005) 513. https://doi.org/10.1016/j. medengphy.2004.11.005

18 D. N. Pamukoff, E. D. Ryan, and J. T. Blackburn: J. Electromyogr. Kinesiol. 24 (2014) 888. https://doi. org/10.1016/j.jelekin.2014.07.014

19 C. Rothmuller and E. Cafarelli: J. Physiol. 485 (1995) 857. https://doi.org/10.1113/jphysiol.1995.sp020775

20 S. Lopez, F. Bini, C. Del-Percio, F. Marinozzi, C. Celletti, A. Suppa, R. Ferri, E. Staltari, F. Camerota, and C. Babiloni: Neuroscience 352 (2017) 236. https://doi.org/10.1016/j.neuroscience.2017.03.015

21 J. S. Schofield, M. R. Dawson, J. P. Carey, and J. S. Hebert: Technol. Health. Care. 23 (2015) 129. https://doi. org/10.3233/THC-140879

22 T. Kodama, H. Nakano, H. Ohsugi, and S. Murata: J. Phys. Ther. Sci. 28 (2016) 419. https://doi.org/10.1589/ jpts. 28.419

23 A. F. Cordero, M. Steyvers, and O. Levin: Behav. Brain. Res. 190 (2008) 41. https://doi.org/10.1016/j. bbr.2008.02.019

24 M. Steyvers, O. Levin, S. M. Verschueren, and S. P. Swinnen: Exp. Brain. Res. 151 (2003) 9. https://doi. org/10.1007/s00221-003-1427-3

25 T. Mima, N. Simpkins, T. Oluwatimilehin, and M. Hallett: Neurosci. Lett. 275 (1999) 77. https://doi.org/10.1016/ s0304-3940(99)00734-x

26 S. Sharif-Bidabadi, I. Murray, and G. Y. F. Lee: Biomed. Eng. Lett. 8 (2018) 283 https://doi.org/10.1007/s13534018-0072-5 
27 H. Hermens, B. Freriks, R. Merletti, D. Stegeman, J. Block, G. Rau, C. Disselhorst-Klug, and G. Hagg, Eds.: SENIAM 8: European Recommendations for Surface ElectroMyoGraphy (Roessingh Research and Development, Enschede, 1999).

28 K. Falconer and D. A. Winter: Electromyogr. Clin. Neurophysiol. 25 (1985) 135.

29 K. Kwak, H. Kim, K. Song, and D. Kim: J. Vibroeng. 19 (2017) 3866. https://doi.org/10.21595/jve.2017.18886

30 K. Kwak, H. Kim, and D. Kim. Biomed: Biomed. Eng. Lett. 6 (2016) 16 https://doi.org/10.1007/s13534-016$\underline{0215-5}$

31 R. S. Johansson and A. B. Vallbo: J. Physiol. 256 (1979) 283 https://doi.org/10.1113/jphysiol.1979.sp012619

32 S. Gilman: J. Neurol. Neurosurg. Psychiatry. 73 (2002) 473 http://dx.doi.org/10.1136/jnnp.73.5.473

33 B. Marconi, G. M. Filippi, G. Koch, C. Pecchioli, S. Salerno, R. Don, F. Camerota, V. M. Saraceni, and C. Caltagirone: J. Neurol. Sci. 275 (2008) 51. https://doi.org/10.1016/j.jns.2008.07.025

34 S. Yoshioka, A. Nagano, D. C. Hay, and S. Fukashiro: Biomed. Eng. Online. 8 (2009) 27. https://doi. org/10.1186/1475-925X-8-27

35 M. Galli, V. Cimolin, M. Crivellini, and I. Campanini: Gait. Posture. 28 (2008) 80. https://doi.org/10.1016/j. gaitpost.2007.10.003

36 M. C. Ridding and J. L. Taylor: J. Physiol. 537 (2001) 623. https://doi.org/10.1111/j.1469-7793.2001.00623.x

37 L. W. Klykken, B. G. Pietrosimone, K. M. Kim, C. D. Ingersoll, and J. Hertel: J. Athl. Train. 46 (2011) 263. https://doi.org/10.4085/1062-6050-46.3.263

38 S. Mari, M. Serrao, C. Casali, C. Conte, G. Martino, A. Ranavolo, G. Coppola, F. Draicchio, L. Padua, G. Sandrini, and F. Pierelli: Cerebellum 13 (2014) 226. https://doi.org/10.1007/s12311-013-0533-4

39 P. A. Guertin: Front. Neurol. 3 (2013) 183 https://doi.org/10.3389/fneur.2012.00183

40 E. R. Kandel, J. H. Schwartz, and T. M. Jessell, Eds.: Principles of Neural Science (McGraw-Hill, New York, 2011) 4th ed., pp. 28-35

41 T. Hortobágyi and P. DeVita: J. Electromyogr. Kinesiol. 10 (2000) 117. https://doi.org/10.1016/S10506411(99)00026-7

42 S. S. Shin and W. G. Yoo: J. Phys. Ther. Sci. 28 (2016) 1922. https://doi.org/10.1589/jpts.28.1922

\section{About the Authors}

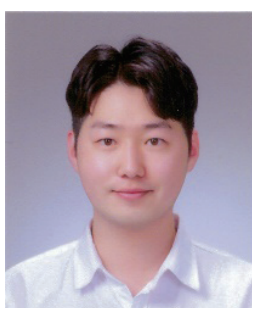

Seunghun Ko received his B.S. degree from Jeonbuk National University, Korea, in 2018 and his M.S. degree from Jeonbuk National University in 2020. His research interests are in biomechanics, biopotential analysis, and elderly gait analysis. (rhtmgnd93@naver.com)

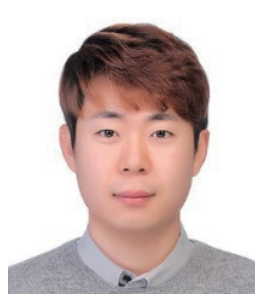

Kiyoung Kwak received his B.S., M.S., and Ph.D. degrees from Jeonbuk National University, Republic of Korea, in 2009, 2011, and 2018, respectively. $\mathrm{He}$ is currently a postdoctoral researcher at the Division of Biomedical Engineering, Jeonbuk National University. His major research interests are in neuro-musculoskeletal biomechanics, sensory-motor integration, rehabilitation engineering, and cognition function in the elderly. (kykwak@jbnu.ac.kr)

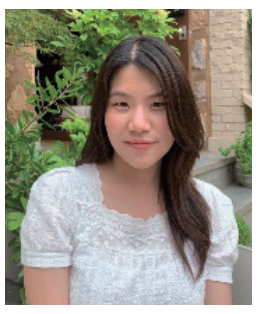

Mattatika Pomrop received her B.S. degree from Chiang-Mai University, Thailand, in 2013 and her M.S. degree from Jeonbuk National University, Republic of Korea, in 2020. From 2013 to 2017, she was a nuclear medicine technician at Bumrungrad International Hospital, Thailand. Her research interests are in muscle physiology, kinesiology, biomechanics, and radiation. (mattatika.p@gmail.com) 


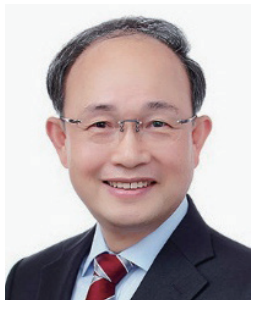

Dongwook Kim received his Ph.D. degree in biomedical engineering from Hokkaido University, Sapporo, Japan, in 1995. He is now a professor at the Division of Biomedical Engineering, Jeonbuk National University, Republic of Korea, and the president of the Korean Society of Medical and Biological Engineering. His current research interests include biomedical engineering, rehabilitation engineering, sensory-motor integration, diagnosis, and healthcare systems. (biomed@jbnu.ac.kr) 